\title{
Consumo alimentar de idosos com doença de Alzheimer
}

\author{
Saulo Victor e Silva*, Francisca Cleice da Silva Soares Miranda**, Samara Costa de Queiroz ${ }^{\star \star \star}$, Juliany \\ Caroline Silva de Sousa ${ }^{\star \star \star}$, Alexandre Coelho Serquiz ${ }^{\star \star \star \star \star}$
}

\section{Resumo}

\begin{abstract}
A Doença de Alzheimer (DA) é o tipo mais comum de demência, acometendo $1 \%$ ou pouco mais de $6 \%$ da população a partir dos 65 anos. A influência dos aspectos nutricionais no processo de envelhecimento e na demência tem sido estudada desde sua participação protetora até a sua possível ação no retardo das disfunções e alterações degenerativas inerentes à idade. $\mathrm{O}$ objetivo do estudo foi analisar o consumo alimentar dos principais grupos alimentares pelos idosos com DA acompanhados no Centro Especializado de Atenção à Saúde do idoso (CEASI) em Natal-RN. Trata-se de um estudo descritivo de abordagem quantitativa com 58 idosos atendidos no CEASI. Para a coleta de dados, foi aplicado um questionário validado semiestruturado com questões fechadas. Os dados foram coletados em setembro de 2014. A média de idade dos idosos foi de 82 anos $( \pm 7,33)$, sendo $57 \%$ do sexo feminino. Nos grupos
\end{abstract}

analisados, as frutas, legumes e verduras, encontram-se incluídos nos hábitos alimentares e possuem boa aceitação, assim como alguns carboidratos, como: cereais integrais e massas. O consumo de tubérculos, frituras, gorduras, embutidos e doces foi considerado baixo. Leite e derivados também tiveram bom consumo, apesar de não ser o hábito de alguns idosos com DA, possivelmente devido à dificuldade de deglutição. Outras condições dificultam a adesão de alguns alimentos, como a redução das papilas gustativas, dificuldade na aceitação das texturas, sabores e consistências dos alimentos. Sendo assim, conseguiu-se analisar qualitativamente e quantitativamente a adesão dos diferentes grupos alimentares dos idosos com DA e sua relação dentro de um contexto fisiológico e cultural.

Palavras chaves: Idoso. Consumo Alimentar. Doença de Alzheimer.

* Nutricionista. Doutorando em Ciências farmacêuticas pela Universidade Federal do Rio Grande do Norte. Mestre em Saúde Coletiva pela Universidade Federal do Rio Grande do Norte, Brasil.

** Nutricionista graduada pela Universidade Potiguar- Laureate International Universities, Natal, Brasil.

*** Nutricionista graduada pela Universidade Potiguar- Laureate International Universities, Natal, Brasil.

**** Nutricionista graduada pela Universidade Potiguar- Laureate International Universities, Natal, Brasil. Rua alberto maranhão,1122 - Tirol, +55 84987415297.

****** Nutricionista. Doutorando do Departamento de Ciências da Saúde da Universidade Federal do Rio Grande do Norte; Mestre em Bioquímica pela Universidade Federal do Rio Grande do Norte, Brasil. Av nascimento de castro, 1050 - Lagoa Nova, +55 849991118

$\rightarrow$ http://dx.doi.org/10.5335/rbceh.v17i1.7609 


\section{Introdução}

No Brasil, o envelhecimento populacional ocorre de modo acelerado, devido ao aumento da expectativa de vida. Prevê-se que a população com 60 anos ou mais, correspondente a $10 \%$ do total em 2010 , atingirá $13,7 \%$ em 2020 , chegando a $23,8 \%$ de idosos em 2040 (MENDES et $a l$., 2012). O envelhecimento populacional trouxe os benefícios de uma maior longevidade, bem como, trouxe um novo perfil de morbimortalidade, caracterizado por um aumento das doenças crônico-degenerativas, como, por exemplo, as demências (NICHOLS et al., 2019).

Dentre as demências, a Doença de Alzheimer (DA) é mais comum, onde cerca de 50 a $60 \%$ destes idosos são acometidos. Acomete $1 \%$ ou pouco mais de $6 \%$ da população a partir dos 65 anos, e atinge valores de prevalência superior a $50 \%$ em indivíduos com 95 anos ou mais (XIMENES; RICO; PEDREIRA, 2014). Dentre os fatores de risco estão: histórico familiar, sexo feminino, idade, dentre outros. Ao ser acometido pela doença, os idosos ficam susceptíveis ao declínio das funções cognitivas e consequentemente, a ação de alimentar-se e escolher os alimentos, ficam comprometidas (FERNANDES; ANDRADE, 2017).

Os pacientes com DA, tendem a apresentar uma perda progressiva de comportamentos aprendidos, incluindo a capacidade de alimentar-se, e frequentemente em alguns ocorre a recusa de alimentos na progressão da doença (PLAGMAN et al., 2019). Mudanças físicas associadas ao processo de enve- lhecimento contribuem com a diminuição do sabor e do odor, reduzindo a alimentação devido à regressão da eficiência de opioides endógenos e aumento da saciedade em decorrência do aumento da sensibilidade ao efeito da colecistoquinina, além da anormalidade de outros neurotransmissores. Níveis cerebrais do neuropeptídeo Y e a norepinefrina, que estimula o apetite, se encontra reduzido nesses pacientes (SNOWDEN et al., 2019). Acredita-se que as diferentes cores e sabores dos alimentos, além de uma dieta adequada nutricionalmente, influenciam no bem-estar físico e mental, no equilíbrio emocional, tratamento e até na prevenção da doença (MARTINS, 2019).

Para os pacientes acometidos pela DA, muitas vezes é necessária uma dieta especial para cada fase da doença, sendo a fase final constituída apenas de dietas líquidas e pastosas. Para isso, o cuidador deve receber orientações nutricionais, afim de oferecer ao paciente o suporte e a assistência nutricional correta. Além disso, se faz importante diminuir a distração do paciente durante as refeições, facilitar a refeição retirando dos alimentos ossos ou cascas, picando ou processando os alimentos, e sempre incrementando com formas e temperos diferentes, incluindo os diferentes grupos alimentares (PEREZ-RAMOS et al., 2016).

Dessa forma, saber quais e quantas vezes os principais grupos alimentares estão inseridos na alimentação desses pacientes é indispensável, tendo em vista que essa avaliação nos mostra o perfil 
nutricional desse público, encorajando para uma possível interferência. Diante do exposto, o estudo tem como objetivo analisar o consumo alimentar por meio dos principais grupos alimentares, de idosos com DA acompanhados no Centro Especializado de Atenção à Saúde do idoso (CEASI) em Natal-RN.

\section{Materiais e métodos}

Trata-se de um estudo descritivo de abordagem quantitativa, observacional transversal. O trabalho foi aprovado pelo comitê de ética em pesquisa da Universidade Potiguar (CAAE: 57817716.5.0000.5296)

A população estudada foi de idosos portadores de DA atendidos no Centro Especializado de Atenção à Saúde do Idoso (CEASI), localizado no município de Natal - RN, com uma amostra de 58 idosos de ambos os sexos, sendo destes, 07 foram incluídos no estudo. Foram utilizados como critérios de inclusão ser idoso com idade igual ou superior a 60 anos, portador de DA, ter capacidade de verbalização oral e que voluntariamente aceitasse participar da pesquisa. Quando sem a capacidade de responder, o cuidador poderia responder, se estivesse exercendo essa função há pelo menos um mês com o mesmo idoso. Foram excluídos da pesquisa os idosos que não possuíam cuidador fixo, ou se os cuidadores eram menores de 18 anos, ou se os idosos não tinham diagnóstico de DA confirmado.

Para coleta dos dados foi realizada uma entrevista com participantes através de um questionário semiestruturado com questões fechadas oriunda do Projeto Saúde, Bem-estar e Envelhecimento na América Latina e Caribe (Projeto SABE), que constitui um estudo epidemiológico transversal, de base populacional domiciliar, sob a coordenação da Organização Pan-Americana da Saúde (OPAS). Os dados foram coletados no período de agosto a setembro do ano de 2014, por estudantes do curso de nutrição da Universidade Potiguar.

Dentre os questionários, investigou-se os aspectos nutricionais, divididos em grupos alimentares (frutas, verduras/ legumes, leguminosas, carne/peixe, ovos, leite/iogurte, massas, cereal integral, tubérculos, frituras, gordura animal, gordura vegetal, enlatados, embutidos, doces/balas, temperos e molhos, além de classificação dos alimentos quanto a característica diet /light).

Realizou-se a análise estatística descritiva dos dados, em seguida a média e porcentagem foi obtida por meio do programa Statistical Package for the Social Science (SPSS) Statistics 20.0, considerando significativo $\mathrm{p}<0,05$.

\section{Resultados e discussão}

A idade média dos idosos foi de 82 anos ( $\pm 7,33)$, a idade mínima de 63 e a máxima de 95 anos. Em relação ao gênero, a maioria foi do sexo feminino (57\%). Quanto à condição sócio econômica, todos os idosos apresentaram renda de até três salários mínimos, que corresponde, em sua maioria, à aposentadoria. Além disso, residem com até três pessoas em seus domicílios. 
Na Tabela 1, observa-se que $60 \%$ $(\mathrm{n}=2)$ faz de 5 a 6 refeições por dia e $81 \%$ $(\mathrm{n}=5)$ dos idosos relatam ter horário certo para se alimentar, e 39\% disseram ter aversão a algum tipo de alimento.

De acordo com a Figura 1, observou-se que $89 \%$ dos idosos consumiam frutas, legumes e verduras, pelo menos 2 a 3 vezes por dia. Machado et al. (2009) em seu estudo sobre o consumo de frutas e verduras de idosos com
DA - maioria do sexo feminino $(65 \%)$ e apresentando DA leve - $85 \%$ não atingiram o recomendado pela Organização Mundial da Saúde (OMS, 2003). É imprescindível que os idosos garantam o aporte de substâncias antioxidantes por meio das frutas e verduras, tendo em vista que o dano oxidativo parece estar envolvido na patogênese da DA (CARDOSO; JACKIX; PIETRO, 2016; CASATI et al., 2020).

Figura 1 - Consumo de legumes e verduras dos idosos com DA do CEASI/RN, 2014

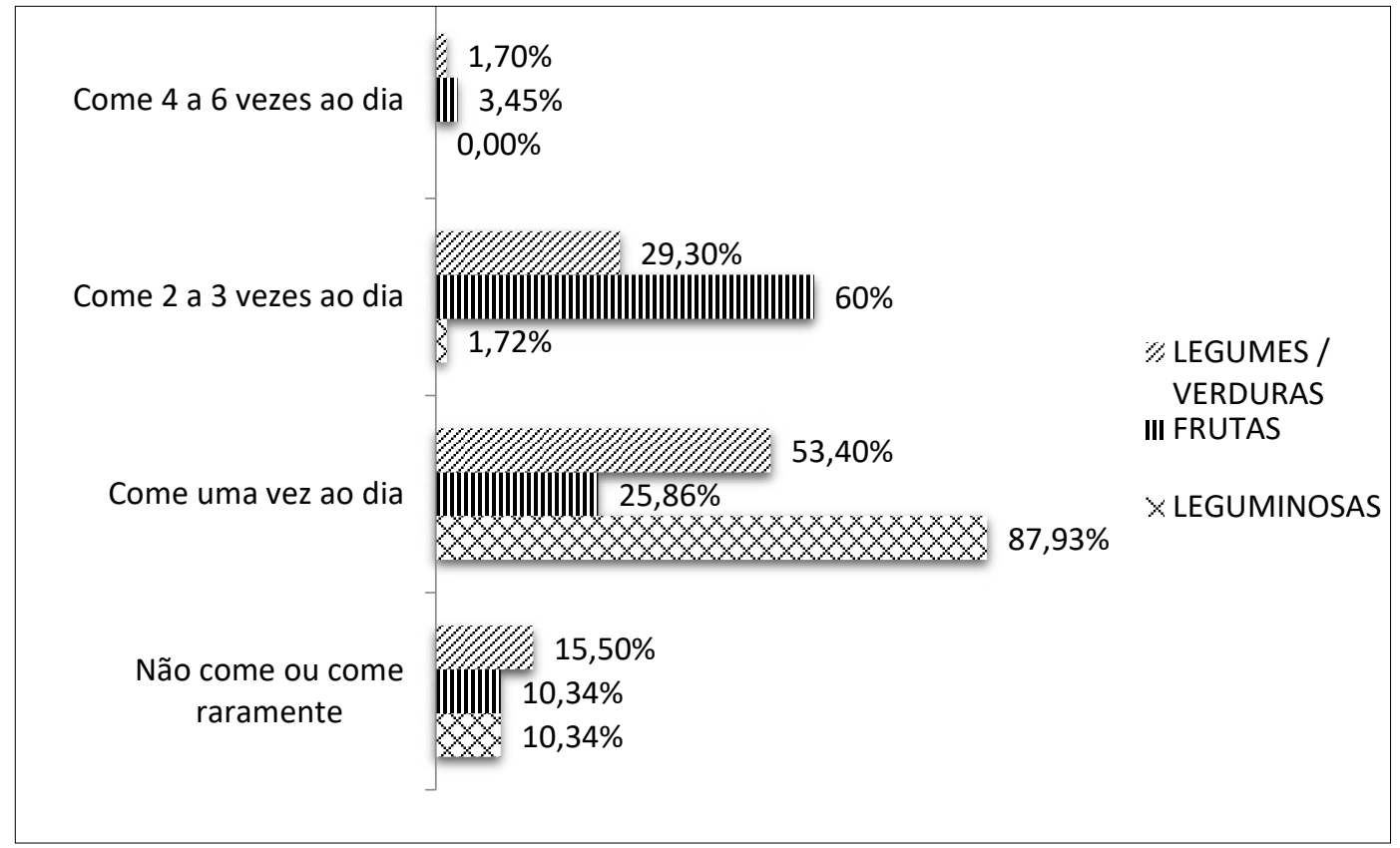

Fonte: Autores (2014).

Ao observar a Figura 2, quanto ao consumo de leite e derivados, percebemos que $48 \%$ dos idosos consomem pelo menos 2 a 3 vezes ao dia, resultado que corrobora com o estudo sobre $o$ alto $(64,4 \%)$ consumo de laticínios nos indi- víduos com 60 anos ou mais (MUNIZ; MADRUGA; ARAÚJO, 2013). Uma pequena parte dos idosos estudados (13\%) não consomem leite e derivados (fontes de cálcio de origem animal). No estudo de Machado et al. (2009) com relação ao 
grupo de leite e derivados em idosos com DA, $13,5 \%$ dos idosos com estágio leve e $28,5 \%$ com estágio moderado referiram nunca ingerir, conforme entrevista com os cuidadores. A carência de cálcio, está associado a problemas ósseos, como a osteoporose e fraturas, principalmente nesta faixa etária (CUESTA-TRIANA et al., 2019). Essa redução do consumo pode relacionar-se a alterações na deglutição, já que a dificuldade se eleva principalmente no consumo de líquidos (DODDERI; KARKERA; SUNIL, 2019)

Em relação ao consumo de carboidratos, pouco mais da metade $(53 \%)$ dos idosos consumiam cereais integrais pelo menos uma vez ao dia. Os cereais integrais, possuem alto teor de fibra, importante para o trânsito intestinal, redução do risco de doenças crônicas e redução de processos inflamatórios de baixo grau (FONTANELLI et al., 2019; YANNAKOULIA et al., 2018). Ao analisar o consumo de massas (carboidratos simples), como pão e arroz branco, foi observado que uma grande parte dos idosos consumia pelo menos 2 a 3 vezes por dia. Esses dados condizem ao levantamento da Pesquisa de Orçamentos Familiares (POF 2002-2003), os quais relatam que na alimentação brasileira, o arroz e o pão apresentam consumo elevado, sendo, respectivamente, o primeiro e o segundo alimento mais consumidos do grupo dos cereais (MIRANDA; PAIVA,
2020; SILVA et al., 2010). No estudo de Machado et al. (2009), 50,3\% a 59,3\% das kcal ingeridas no dia, eram provenientes dos carboidratos na dieta de idosos com DA leve e moderada, respectivamente, considerado um consumo adequado segundo o Consumo Dietético de Referência (PADOVANI et al., 2006). Além disso, o estudo mostrava que maior parte dos idosos com DA leve e moderada (91\% e $61 \%$ ), respectivamente, consumiam diariamente alimentos do grupo dos cereais. Mendes et al. (2016), observou que quando comparado o grupo controle, os idosos com DA apresentaram um menor consumo de carboidratos, com exceção daqueles que faziam uso de espessantes.

Entre os tubérculos, a macaxeira e a batata doce foram os carboidratos de maior preferência entre os idosos, entretanto o consumo não foi considerado parte do hábito alimentar. A consistência e/ou sabor, pode estar ligada a aceitação, tendo em vista que, esse grupo alimentar possui textura mais consistente e sabor menos acentuado, principalmente para os idosos que possuem perda das papilas gustativas (PIERONI et al., 2016). A forma com que é realizada a preparação é fundamental, tanto para aceitação, quanto para não ocorrências de engasgos relacionado a alimentação. 
Figura 2 - Consumo de Massas, Cereal integral e tubérculos dos idosos com DA do CEASI/RN, 2014

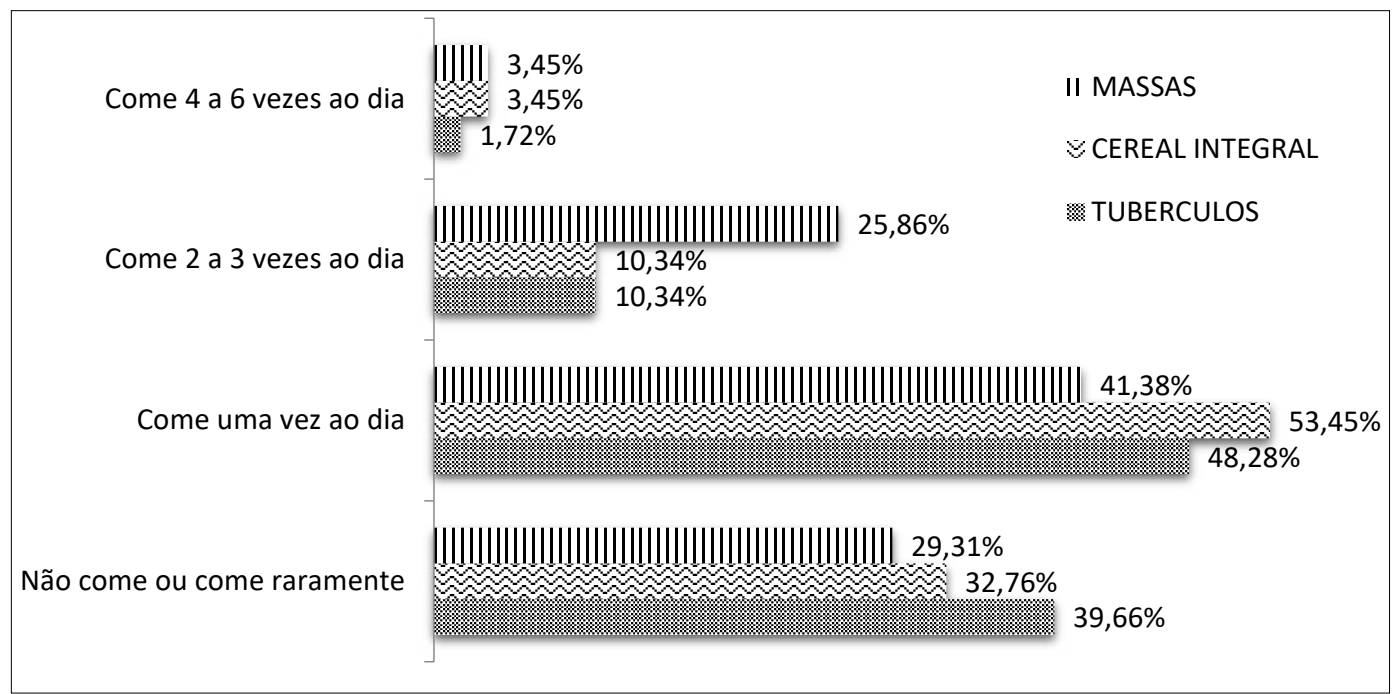

Fonte: Autores (2014).

Na Figura 3, o consumo de carnes e peixes foi diário (69\%), diferente do consumo de ovos que teve um reduzido consumo ( $24 \%$ comiam diariamente), corroborando com o estudo de Machado et al (2009), onde o ovo foi o alimento que mostrou menor frequência de consumo diário pelos idosos, citado como o alimento ingerido apenas uma vez por semana ou mesmo nunca sendo ingerido. Possivelmente, devido à dificuldade de deglutição e palatabilidade quando não inserido temperos naturais para agregar sabor (HOPPU; PUPUTTI; SHANDELL, 2020). Ainda no estudo de Machado et al. (2009), no grupo das carnes, mais de $50 \%$ dos idosos com DA também as consumiam diariamente, sendo principalmente as carnes vermelhas. Esse grupo alimentar, pode ser inserido na alimentação por meio de diferentes técnicas dietéticas, podendo ser melhor aceito por esses pacientes. Carnes vermelhas é uma boa fonte de vitamina B12 - idosos podem encontrar-se com diminuição na absorção dessa vitamina pela interação fármaco-nutriente e acloridria, por exemplo, além da sua relação com alterações neurológicas e o desenvolvimento de demência e DA, quando em níveis deficientes (BOSTON et al., 2020; SANTOS; PARDI, 2020). E, os peixes, principalmente aqueles de águas frias $\mathrm{e}$ profundas, possuem boa quantidade de ácido docosaexaenoico (DHA) (CHIU; VENKATAKRISHNAN; WANG, 2020; TOMASZEWSKI et al., 2020, XIE et al., 2020), importante por reduzir o risco de demência atuando na redução da inflamação a nível celular (RAJI et al., 2014). 
Figura 3 - Consumo de carne/peixe e ovos dos idosos com DA do CEASI/RN, 2014

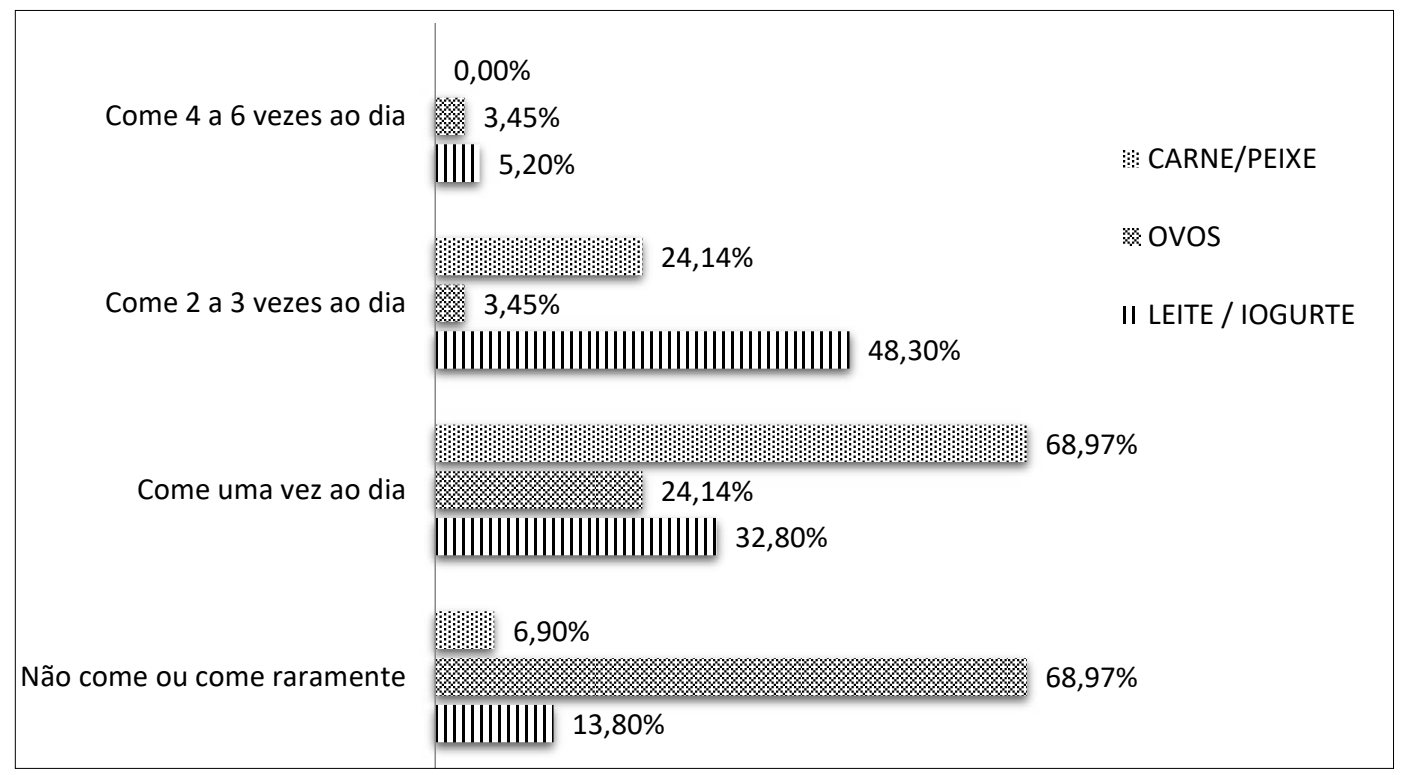

Fonte: Dos autores (2014).

Quanto às frituras e gorduras animal (Figura 4) a frequência foi baixa e muitas vezes inexistente do consumo alimentar. A gordura vegetal foi consumida $1 \mathrm{vez}$ ao dia pela maioria dos idosos, enquadrando-se em consumo diário. As gorduras são fontes de energia para o organismo, porém, é importante distinguir as que são saudáveis e essenciais, daquelas que devem ser evitadas por prejudicar a saúde, como as gorduras saturadas em excesso e as gorduras trans (KAO et al., 2020). Desde que utilizados com moderação em preparações culinárias com base em alimentos in natura ou minimamente processados, os óleos e as gorduras, contribuem para diversificar e tornar mais saborosa a alimentação sem que fique nutricionalmente desbalanceada. Sendo assim, quando consumidas mesmo que diariamente no preparo dos alimentos e em quantidades adequadas, não trazem prejuízos à saúde (BRASIL, 2004; FONTEH, et al. 2020). 
Figura 4 - Consumo de frutas, gordura animal e vegetal dos idosos com DA do CEASI/RN, 2014

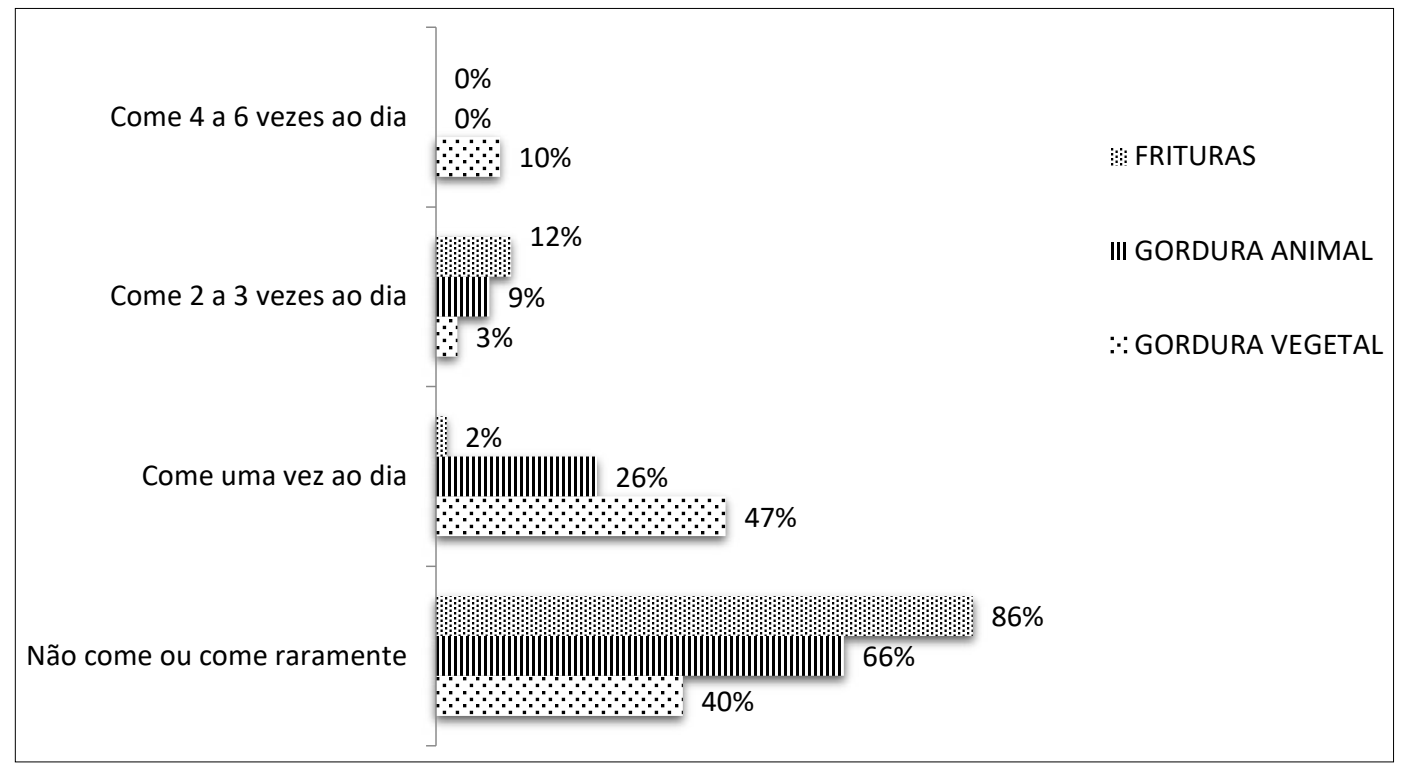

Fonte: Autores (2014).

O consumo de enlatados, embutidos, doces e balas na Figura 5, apresentou-se quase nulo, corroborando com o estudo de Machado et al. (2009) onde o consumo de doces em geral revelou elevada recusa e ausência na dieta consumida pelos idosos pertencentes ao estágio moderado de DA. Todavia, os molhos e temperos industrializados ainda mostraram consumo significativo diário por parte dos idosos. No estudo de Almeida et al. (2018), quando aplicado também o estudo para idosos do estudo SABE (Saúde, Bem-Estar e Envelhecimento), observou-se que cerca de metade da população estudada relatou não consumir embutidos $(54,2 \%)$ e enlatados $(48,4 \%)$, com apenas $4 \%$ e $3,5 \%$ apresentando alto consumo destes produtos, respec- tivamente. Em relação ao consumo de doces e o uso de temperos ou molhos industrializados, foi notado nesse estudo, que embora uma significativa parcela dos idosos refira não consumir estes produtos (41\%, no caso dos doces e $58,7 \%$, no caso dos temperos ou molhos), $26,1 \%$ e $20,6 \%$ referiram alto consumo destes alimentos, respectivamente. Este consumo pode ser reflexo da perda gustativa no paladar desta faixa etária, gerando uma maior tendência a acrescentar temperos e condimentos com a finalidade de realçar sabor aos alimentos. É bastante usual as pessoas com demência apreciar sabores que nunca gostaram antes ou já não gostarem de alimentos que sempre comeram (CARDOSO; PAIVA, 2017). 
Figura 5 - Consumo de enlatados, embutidos, doces e balas dos idosos com DA do CEASI/RN, 2014

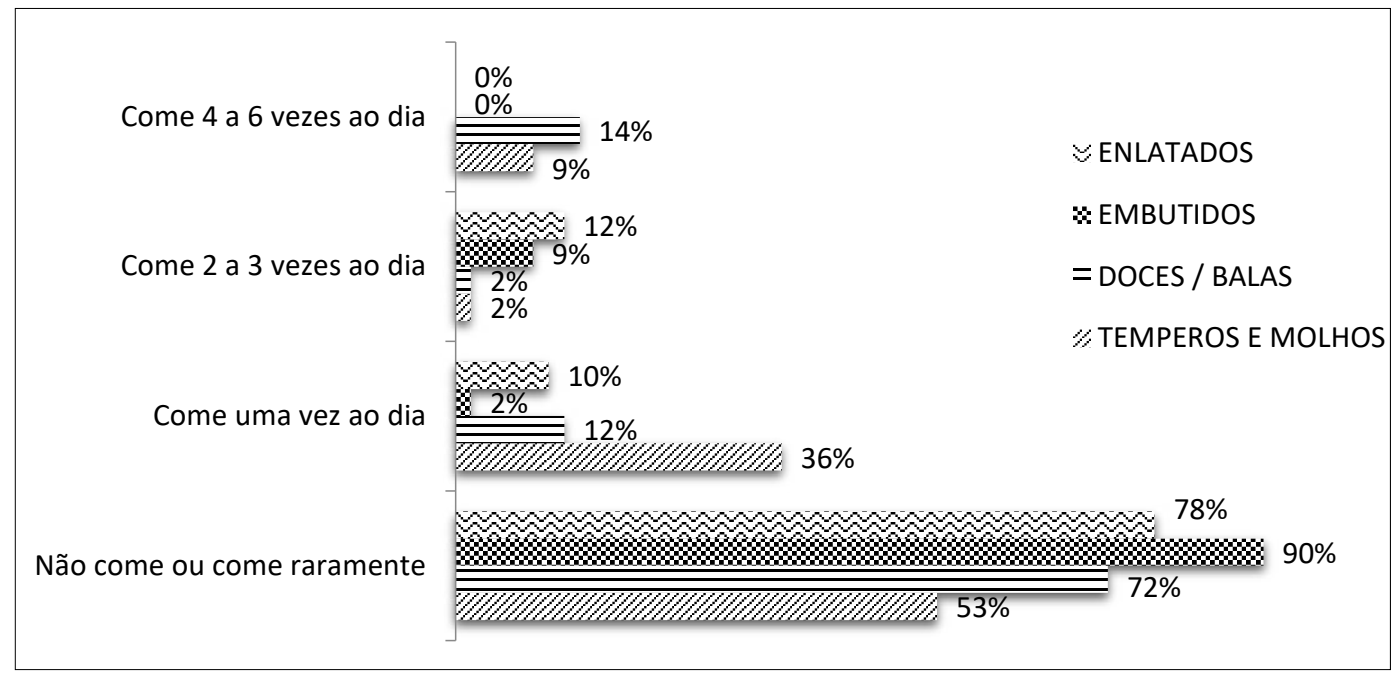

Fonte: Autores (2014).

Quanto aos alimentos diet e light (Figura 6), foi observado também o baixo consumo na alimentação dos idosos corroborando com o estudo de e Almeida et al (2018), onde a maioria dos idosos com DA não consumiam esse tipo de alimento. Uma possível explicação para o baixo consumo desses alimentos é a renda financeira, já que de acordo com o público, a inclusão desses produtos na dieta apresenta custos mais elevados, gerando mais dificuldade de adesão, consequentemente, seu consumo habitual (RODRÍGUEZ DÍAZ, 2020; ALMEIDA et al., 2011).

Em nosso estudo, as limitações estão relacionadas especialmente quanto aos estágios de DA apresentados pelos pacientes participantes.

Figura 6 - Consumo de Diet/Light dos idosos com DA do CEASI/RN, 2014

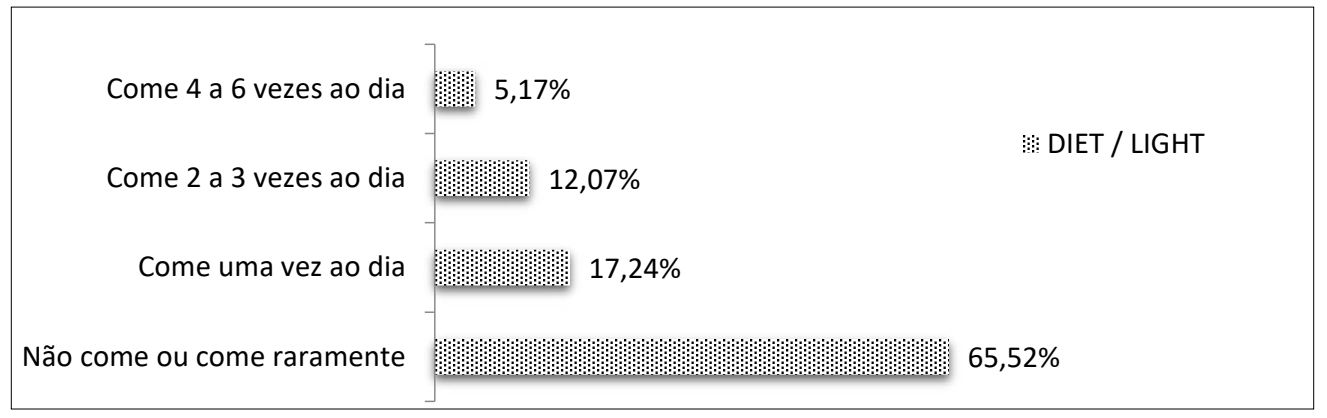

Fonte: Autores (2014). 


\section{Considerações Finais}

Os idosos com DA, bem como seus, cuidadores demonstram, preocupação nas escolhas e seleção dos alimentos. Nos grupos analisados, as frutas, legumes e verduras, encontram-se incluídos nos hábitos alimentares e possuem boa aceitação, assim como alguns carboidratos, como: cereais integrais e massas. O consumo de tubérculos, frituras, gorduras, embutidos e doces foi considerado baixo. Leite e derivados também tiveram bom consumo, apesar de não ser o hábito de alguns idosos com DA, possivelmente devido à dificuldade de deglutição. Outras condições dificultam a adesão, como a redução das papilas gustativas, dificuldade na aceitação das texturas, sabores e consistências dos alimentos.

A orientação nutricional se faz necessária para melhor aceitabilidade, principalmente quanto a técnica dietética a ser utilizada e combinações a serem feitas.

\section{Food consumption of elderly people with Alzheimer's Disease}

\section{Abstract}

Alzheimer's disease (AD) is the most common type of dementia, affecting $1 \%$ or just over $6 \%$ of the population aged 65 and over. The influence of nutritional aspects on the aging process and on dementia was studied from its protected participation to its possible action in delaying changes and degenerative changes inherent to age. The aim of the study was to analyze the food consumption of the main food groups for the elderly with AD at the Specialized Center for Elderly Health Care (CEASI) in Natal-RN. This is a descriptive study with a quantitative approach with 58 elderly people treated at CEASI. For data collection, a validated semi-structured questionnaire with closed questions was applied. Data were collected in September 2014. The average age of the elderly was 82 years old ( \pm 7.33$)$, with $57 \%$ being female. In the groups analyzed, such as fruits and vegetables, the foods included and the foods are good, as well as some carbohydrates, such as: whole grains and pasta. The consumption of tubers, fried foods, fats, sausages and sweets was considered low. Milk and dairy products also had a good consumption, although they are not subject to some elderly people with $\mathrm{AD}$, possibly due to the difficulty in swallowing. Other conditions hinder the adherence of some foods, such as the reduction of taste buds, difficulty in accepting textures, flavors and consistencies of food. Thus, it was able to analyze qualitatively and quantitatively the adherence of different food groups of elderly people with $\mathrm{AD}$ and their relationship within a physiological and cultural context.

Keywords: Elderly. Food Consumption. Alzheimer's disease. 
Nota

1 Local onde o estudo foi realizado: Centro Especializado de Atenção à Saúde do Idoso - R. Augusto Severo, 281 - Ribeira, Natal - RN, 59012-380.

\section{Referências}

ALMEIDA, G. P; ROESIGER, M.A; DOURADO, D.A.Q.S; LIMA, S.S. Viver Sozinho e Consumo de Ultraprocessados por Idosos do Estudo Sabe (Saúde, Bem-Estar e Envelhecimento). International Journal of Nutrology, v. 11, n. S 01, p. 820, 2018. DOI: 10.1055/s-0038-1675117.

ALMEIDA, I. C; GUIMARÃES, G.F; REZENDE, D.C; SETTE, R.S. Hábitos alimentares da população idosa: padrões de compra e consumo. Agroalimentaria, v. 17, n. 33, p. 95-110, 2011. DOI: 199220006008

BOSTON, P. F; MCKIRDY, S.J; AL-TURKI, M.A; BAKER, M.E; RUSSEL, J.M. Vitamin $\mathrm{B} 12$ and folate levels in progression of Alzheimer's disease-a short report. International Journal of Psychiatry in Clinical Practice, v. 24, n. 1, p. 68-70, 2020. DOI: 10.1080/13651501.2019.1681457.

BRASIL. Pesquisa de Orçamentos Familiares 2002-2003: Primeiros resultados. IBGE. 2 ed. Rio de Janeiro, 2004. Disponível em <https://biblioteca.ibge.gov.br/visualizacao/livros/liv81847.pdf> Acessado em 30 de Maio de 2020.

CARDOSO, J. F., JACKIX, E. A.; PIETRO, L. O Papel dos polifenois na Doença de Alzheimer: revisão sistemática. Journal of the Health Sciences Institute, v. 34, n.4, p. 240-245, 2016. Disponível em: https:// pesquisa.bvsalud.org/portal/resource/pt/ biblio-848942

CARDOSO, S. A.; PAIVA, I. Nutrição e Alimentação na prevenção e terapêutica da Demência. Acta Portuguesa de Nutrição, n. 11, p. 30-34, 2017. Disponível em: http://www. scielo.mec.pt/scielo.php?script=sci_arttext\&pid=S2183-59852017000400005\&lang=pt

CASATI, M; BOCCARDI, V; FERRI, E; BERTAGNOLI, L; BASTIANI, P; CICCONE,S; MANSI, M; SCAMOSCI, M; ROSSI, P.D; MECOCCI,P; AROSIO, B. Vitamin $\mathrm{E}$ and Alzheimer's disease: the mediating role of cellular aging. Aging clinical and experimental research, v. 32 , n.3, p. 459464, 2020. DOI: $10.1007 / \mathrm{s} 40520-019-01209-3$

CHIU, H. F.; VENKATAKRISHNAN, K.; WANG, C. K. The role of nutraceuticals as a complementary therapy against various neurodegenerative diseases: A mini-review. Journal of Traditional and Complementary Medicine, 2020. DOI: https://doi. org/10.1016/j.jtcme.2020.03.008

CUESTA-TRIANA, F; VERDEJO-BRAVO, C; FERNÁNDEZ-PÉREZ, C; MARTÍN-SÁNCHEZ, F.C et al. Effect of milk and other dairy products on the risk of frailty, sarcopenia, and cognitive performance decline in the elderly: A systematic review. Advances in Nutrition, v. 10, n. suppl. 2, p. S105-S119, 2019. DOI: $10.1093 /$ advances/nmy105

DODDERI, T., KARKERA N.O., SUNIL N. Swallowing thin liquids from the rim of the cup and through the straw in healthy geriatrics. Journal of Natural Science, Biology and Medicine, v. 10, n. 1, p. 16, 2019. DOI: 10.4103/jnsbm.JNSBM_167_18

FERNANDES, J. S. G.; ANDRADE, M. S. Revisão sobre a doença de alzheimer: diagnóstico, evolução e cuidados. Psicologia, Saúde \& Doenças, v. 18, n. 1, p. 131-140, 2017. DOI: http://dx.doi.org/10.15309/17psd180111

FONTANELLI, M. M; MICHA, R; SALES, C.H; LIU, J; MOZAFFARIAN, D; FISBERG, R.M. Application of the $\leq 10$ : 1 carbohydrate to fiber ratio to identify healthy grain foods and its association with cardiometabolic risk factors. European Journal of Nutrition, p. 1-11, 2019. DOI: 10.1007/s00394-01902165-4 
FONTEH, A. N; CIPOLLA, M; CHIANG, A.J; EDMINSTER, S.P; ARAKAKI, X; HARRINGTON, M.G. Polyunsaturated Fatty Acid Composition of Cerebrospinal Fluid Fractions Shows Their Contribution to Cognitive Resilience of a Pre-symptomatic Alzheimer's Disease Cohort. Frontiers in physiology, v. 11, p. 83, 2020. DOI: 10.3389 / fphys.2020.00083

HOPPU, U.; PUPUTTI, S.; SANDELL, M. Factors related to sensory properties and consumer acceptance of vegetables. Critical Reviews in Food Science and Nutrition, p. 1-11, 2020. DOI: $10.1080 / 10408398.2020 .1767034$

KAO Y. C; HO, P.C; JOU, I.M; TSAI, K.J. Lipids and Alzheimer's Disease. International journal of molecular sciences, vol. 21, n.4, p. 1505 , 2020. DOI: $10.3390 /$ ijms 21041505

MACHADO, J; CARAM, C.L.B; FRANK, A.A; SOARES, E.A; LAKS, J. Estado nutricional na doença de Alzheimer. Rev. da Associação Médica Brasileira. v.55, n.2, pp.188191, 2009. DOI: https://doi.org/10.1590/ S0104-42302009000200024.

MARTINS, I. Appetite Regulation and the Peripheral Sink Amyloid beta Clearance Pathway in Diabetes and Alzheimer's Disease. In: Top 10 commentaries in Alzheimer's Disease. Avid Science, p. 2-11, 2019. Disponível em: pi.research-repository.uwa.edu. au/portalfiles/portal/45208423/Appetite_Regulation_and_the_Peripheral_Sink_Amyloid_beta_Clearance_Pathway_in_Diabetes_and_Alzheimer_s_Disease.pdf

MENDES A. C. D. et al. Assistência pública de saúde no contexto da transição demográfica brasileira: exigências atuais e futuras. Cad. Saúde Pública, v. 28, n. 5, p. 955-964, 2012. DOI: http://dx.doi.org/10.1590/S0102311X2012000500014.

MENDES, L. P. et al. Avaliação do estado nutricional e consumo alimentar em pacientes com Doença de Alzheimer. Rev. Universidade Vale do Rio Verde, v. 14, n. 2, p. 502-515, 2016. DOI: http://dx.doi.org/10.5892/ruvrd. v14i2.2638
MIRANDA, R. N. A.; PAIVA, M. B. Antropometria e consumo alimentar: identificador do estado nutricional de idosos. Nutrição Brasil, v. 18, n. 3, p. 141-150, 2020. DOI: 10.33233/nb.v18i3.2839

MUNIZ L. C., MADRUGA, S. W., ARAÚJO, C. L. Consumo de leite e derivados entre adultos e idosos no Sul do Brasil: um estudo de base populacional. Rev. Ciência \& Saúde Coletiva, v.18, n.12, 2013. DOI: https:// doi.org/10.1590/S1413-81232013001200008.

NICHOLS, E. et al. Carga global, regional e nacional da doença de Alzheimer e outras demências, 1990-2016: uma análise sistemática para o Estudo Global de Carga de Doenças 2016. The Lancet Neurology, v. 392, n. 10149, p. 760-775, 2018. DOI: https:// doi.org/10.1016/S0140-6736(18)31221-2

ORGANIZAÇÃO MUNDIAL DE SAÚDE. The World Health Report 2003: Shaping the Future. 2003. Disponível em <http:// www.who.int/whr/2003>. Acessado em 01 de Junho de 2020.

PADOVANI, R. M. et al. Dietary reference intakes: aplicabilidade das tabelas em estudos nutricionais. Revista de Nutrição, v. 19, n. 6, p. 741-760, 2006. DOI: http://dx.doi. org/10.1590/S1415-52732006000600010

PEREZ-RAMOS, I. C. S. et al. Percepção dos cuidadores sobre as alterações de deglutição causadas pela demência. Rev Bras Ciências da Saúde, v.20, n.2, p.127-132, 2016. DOI: 10.4034/RBCS.2016.20.02.06

PIERONI, D. C. B. et al. Sensibilidade gustativa e estado nutricional de idosas participantes de um Centro Municipal de Atividades para Idosos, Curitiba-PR. Mundo saúde (Impr.), v. 41, n. 2, p. 203-211, 2017. DOI: 10.15343/0104-7809.20174102203211

PLAGMAN, A. et al. Cholecystokinin and Alzheimer's disease: a biomarker of metabolic function, neural integrity, and cognitive performance. Neurobiology of aging, v. 76, p. 201-207, 2019. DOI: 10.1016/j.neurobiolaging.2019.01.002. 
RAJI, C. A. et al. Regular Fish Consumption and Age-Related Brain Gray Matter Loss. American Journal of Preventive Medicine, vol. 47, n.4, p.444-51, 2014. DOI: 10.1016/j.amepre.2014.05.037.

RODRÍGUEZ DÍAZ, R. R. Análise da elasticidade renda da despesa no Brasil: o caso dos produtos normais, light/diet e orgânicos. Dissertação (Graduação) - Faculdade da Integração Latino - Americana, Foz do Iguaçu, 2020. Disponível em: https://dspace. unila.edu.br/123456789/5419.

SANTOS, G. A. A.; PARDI, P. C. Biomarkers in Alzheimer's disease: Evaluation of platelets, hemoglobin and vitamin B12. Dementia \& Neuropsychologia, v. 14, n. 1, p. 35-40, 2020. DOI: https://doi.org/10.1590/ 1980-57642020dn14-010006.

SILVA, A. K. Q. et al. Perfil nutricional de idosos assistidos em instituição de longa permanência na cidade de Natal, RN. Geriatrics, Gerontology and Aging, v. 4, n. 1, p. 27-35, 2010. Disponível em: http://ggaging. com/details/295/pt-BR/perfil-nutricional-de-idosos-assistidos-em-instituicao-de-longa-permanencia-na-cidade-de-natal--rn.

SNOWDEN, S. G. et al. Neurotransmitter Imbalance in the Brain and Alzheimer's Disease Pathology. Journal of Alzheimer's Disease, n. Preprint, p. 1-9, 2019. DOI: 10.3233/JAD-190577.

TOMASZEWSKI, N. et al. Effect of APOE Genotype on Plasma Docosahexaenoic Acid (DHA), Eicosapentaenoic Acid, Arachidonic Acid, and Hippocampal Volume in the Alzheimer's Disease Cooperative StudySponsored DHA Clinical Trial. Journal of Alzheimer's Disease, n. Preprint, p. 1-16, 2020. DOI: 10.3233/JAD-191017.

XIE, Y. et al. Fish oil protects the blood-brain barrier integrity in a mouse model of Alzheimer's disease. Chinese Medicine, v. 15, p. 1-11, 2020. DOI: https://doi.org/10.1186/ s13020-020-00314-0.
XIMENES, M. A., RICO, B. L. D., PEDREIRA, R. Q. Doença de Alzheimer: a dependência e o cuidado. Rev Kairós Gerontologia, v.17, n.2, p.121-140, 2014. DOI: https://doi. org/10.23925/2176-901X.2014v17i2p121-140.

YANNAKOULIA, M. et al. Eating habits and behaviors of older people: Where are we now and where should we go? Maturitas, v. 114, p. 14-21, 2018. DOI: 10.1016/j.maturitas.2018.05.001.

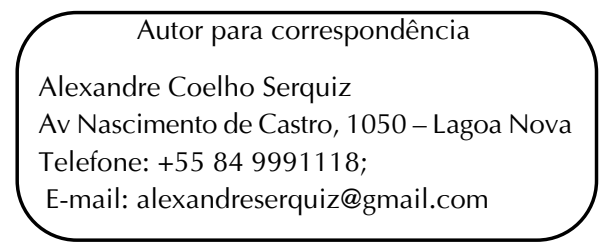

